Conf $950226--39$

UCRL-JC-120215

PREPRINT

\title{
Plasma Mediated Ablation of Biological Tissues with Ultrashort Laser Pulses
}

\author{
A.A. Oraevsky*, L.B. DaSilva, M.D. Feit, M.E. Glinsky, \\ B.M. Mammini, K.L. Paquette, M.D. Perry, A.M. Rubenchik, \\ W. Small IV, and B.C. Stuart
}

This paper was prepared for submittal to the SPIE's 1995 International Symposium on Lasers and Applications/Biomedical Optics

Laser-Tissue Interaction VI, San Jose, CA

February 4-10, 1995

March 8, 1995

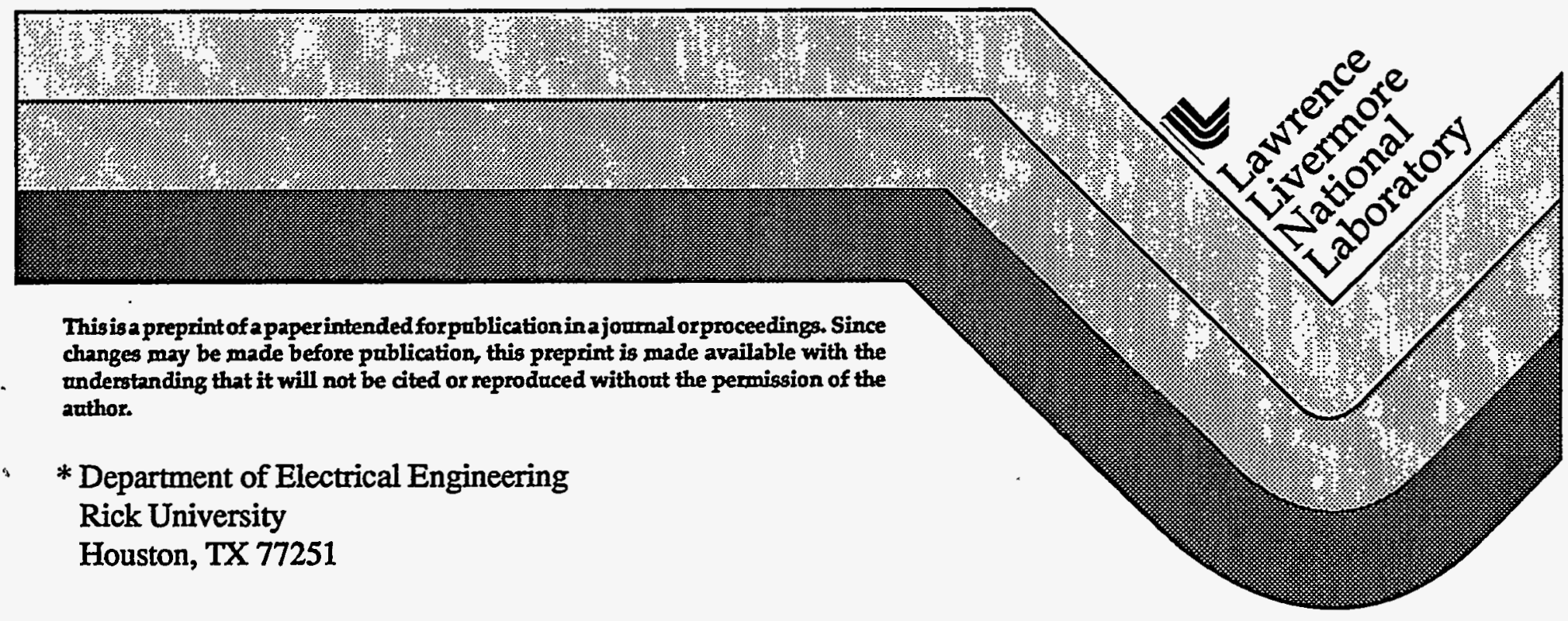




\section{DISCLAIMER}

This document was prepared as an account of work sponsored by an agency of the United States Government. Neither the United States Government nor the University of California nor any of their employees, makes any warranty, express or implied, or assumes any legal liability or responsibility for the accuracy, completeness, or usefulness of any information, apparatus, product, or process disclosed, or represents that its use would not infringe privately owned rights. Reference herein to any specific commercial product, process, or service by trade name, trademark, manufacturer, or otherwise, does not necessarily constitute or imply its endorsement, recommendation, or favoring by the United States Government or the University of California. The views and opinions of authors expressed herein do not necessarily state or reflect those of the United States Government or the University of California, and shall not be used for advertising or product endorsement purposes. 


\section{DISCLAIMER}

Portions of this document may be illegible in electronic image products. Images are produced from the best available original document. 


\title{
Plasma mediated ablation of biological tissues with ultrashort laser pulses
}

\author{
A.A. Oraevsky*, L.B. DaSilva, M.D. Feit, M.E. Glinsky, B.M. Mammini, \\ K.L. Paquette, M.D. Perry, A.M. Rubenchik, W. Small IV, B.C. Stuart \\ Lawrence Livermore National Laboratory, Livermore, CA 94550 \\ *Department of Electrical Engineering, Rice University, Houston, TX 77251
}

\begin{abstract}
Plasma mediated ablation of collagen gels and porcine cornea was studied at various laser pulse durations in the range from $350 \mathrm{fs}$ to $1 \mathrm{~ns}$ at $1053 \mathrm{~nm}$ wavelength. A time resolved stress detection technique was employed to measure transient stress profiles and amplitudes. Optical microscopy was used to characterize ablation craters qualitatively, while a wide band acoustic transducer helped to quantify tissue mechanical response and the ablation threshold. The ablation threshold was measured as a function of laser pulse duration and linear absorption coefficient. For nanosecond pulses the ablation threshold was found to have a strong dependence on the linear absorption coefficient of the material. As the pulse length decreased into the subpicosecond regime the ablation threshold became insensitive to the linear absorption coefficient. The ablation efficiency was found to be insensitive to both the laser pulse duration and the linear absorption coefficient. High quality ablation craters with no thermal or mechanical damage to surrounding material were obtained with $350 \mathrm{fs}$ laser pulses. The mechanism of optical breakdown at the tissue surface was theoretically investigated. In the nanosecond regime, optical breakdown proceeds as an electron collisional avalanche ionization initiated by thermal seed electrons. These seed electrons are created by heating of the tissue by linear absorption. In the ultrashort pulse range, optical breakdown is initiated by the multiphoton ionization of the irradiated medium ( 6 photons in case of tissue irradiated at $1053 \mathrm{~nm}$ wavelength), and becomes less sensitive to the linear absorption coefficient. The energy deposition profile is insensitive to both the laser pulse duration and the linear absorption coefficient.
\end{abstract}

Keywords: optical breakdown, laser ablation, ultrashort pulse, stress waves, recoil momentum, collateral damage.

\section{INTRODUCTION}

Plasma optical breakdown is the only mechanism of laser energy deposition in transparent tissues. Therefore, plasma mediated ablation has been studied by a number of groups ${ }^{1-6}$. It was found that picosecond and subpicosecond ablation produces much lower collateral damage to adjacent tissues than nanosecond ablation. For nanosecond pulse durations, optical breakdown is determined by laser heating of absorbing impurities at the surface of the irradiated medium. This heating produces the seed electrons that ionize the material via the subsequent process of electron collisional avalanche. As one enters the picosecond pulse regime, another mechanism of producing the seed electrons becomes dominate - multiphoton ionization. As a result, the ablation threshold shows less statistical variation. Instead of one seed electron being freed from the tail of the thermal distribution, several photons together ionize the seed electrons. For even shorter pulses, subpicosecond, the multiphoton ionization produces so many electrons that there is little need for the subsequent collisional avalanche. Multiphoton ionization alone becomes the dominate process. This view is supported by our previous studies of plasma mediated damage of transparent dielectrics ${ }^{7}$. This paper presents quantitative experimental and theoretical studies of tissue ablation parameters as functions of pulse duration in the range from 350 fs to 1 ns. Our intention was to observe the difference in tissue response due to the difference in optical breakdown mechanisms while irradiating with various pulse. durations and fluences. The theory of optical breakdown at the surface of biological tissue is not yet developed and the mechanisms underlying plasma mediated ablation are not thoroughly discussed in the literature. Therefore, the emphasis of our study was put on the theoretical description of optical breakdown and the experimental quantification of ablation parameters such as threshold laser fluence $\left(\Phi_{t h}\left[\mathrm{~J} / \mathrm{cm}^{2}\right]\right)$, ablation efficiency $(\eta[\mu \mathrm{m} / \mathrm{pulse}])$, and stress wave amplitude $\left(\sigma_{r}\right.$ [bar]). We have also qualitatively characterized crater quality and collateral damage with the aid of optical microscopy for nanosecond, picosecond and femtosecond ablation.

\section{DISTRIBUTION OF THIS DOCUMENT IS UNLIMITED




\section{MATERIALS AND METHODS}

\subsection{Collagen gels and porcine corneas}

Ten percent collagen gels were made of gelatin G2625 (Sigma, St. Louis, MO) mixed with hot distilled water at $70^{\circ} \mathrm{C}$ and then cooled down to room temperature. Aqueous solutions of cupric chloride $\left(\mathrm{CuCl}_{2}\right)$ with low, medium and high concentrations were used to produce phantom tissues with low $\left(\mu_{a}=0.01 / \mathrm{cm}\right)$, medium $\left(\mu_{a}=22 / \mathrm{cm}\right)$ and high $\left(\mu_{a}=76 / \mathrm{cm}\right)$ absorbency at $1053 \mathrm{~nm}$. To produce gels with extremely high absorbency $\left(\mu_{a} \geq 1000 / \mathrm{cm}\right)$, carbon black micropowder was added to initially transparent liquid gels before hardening. Thin slabs of gels with dimensions of $25 \mathrm{~mm} \times 25 \mathrm{~mm} \times 1.9$ $\mathrm{mm}$ were prepared for the experiments.

Porcine eyes were obtained from a slaughter house, kept on ice during transportation and used for the experiments within two hours. Corneas were not removed from the eye balls for the experiments. Eyes were fixed in a specially designed holder, and the front surfaces of the comeas were irradiated by the laser pulses.

\subsection{Laser system with tunable pulse length}

For plasma mediated ablation studies, we used laser pulses generated by a $1053 \mathrm{~nm}$ Ti:sapphire CPA system ${ }^{8}$ as diagrammed in Fig. 1. Seed pulses of 100 fs from a Kerr-lens mode-locked, Ti:sapphire oscillator were stretched to $1 \mathrm{~ns}$ in a four-pass, single grating (1740 line/mm) pulse stretcher. Amplification by nearly $10^{9}$ to the $6 \mathrm{~mJ}$ range was achieved in the $\mathrm{TEM}_{00}$ stable cavity mode of a linear regenerative amplifier. Further amplification to the $60 \mathrm{~mJ}$ level was achieved in a Ti:sapphire ring regenerative amplifier, which supported a larger $(2.3 \mathrm{~mm})$ beam diameter and reduced nonlinear effects. This system operated at $10 \mathrm{~Hz}$; however, single pulses could be extracted for experiments.

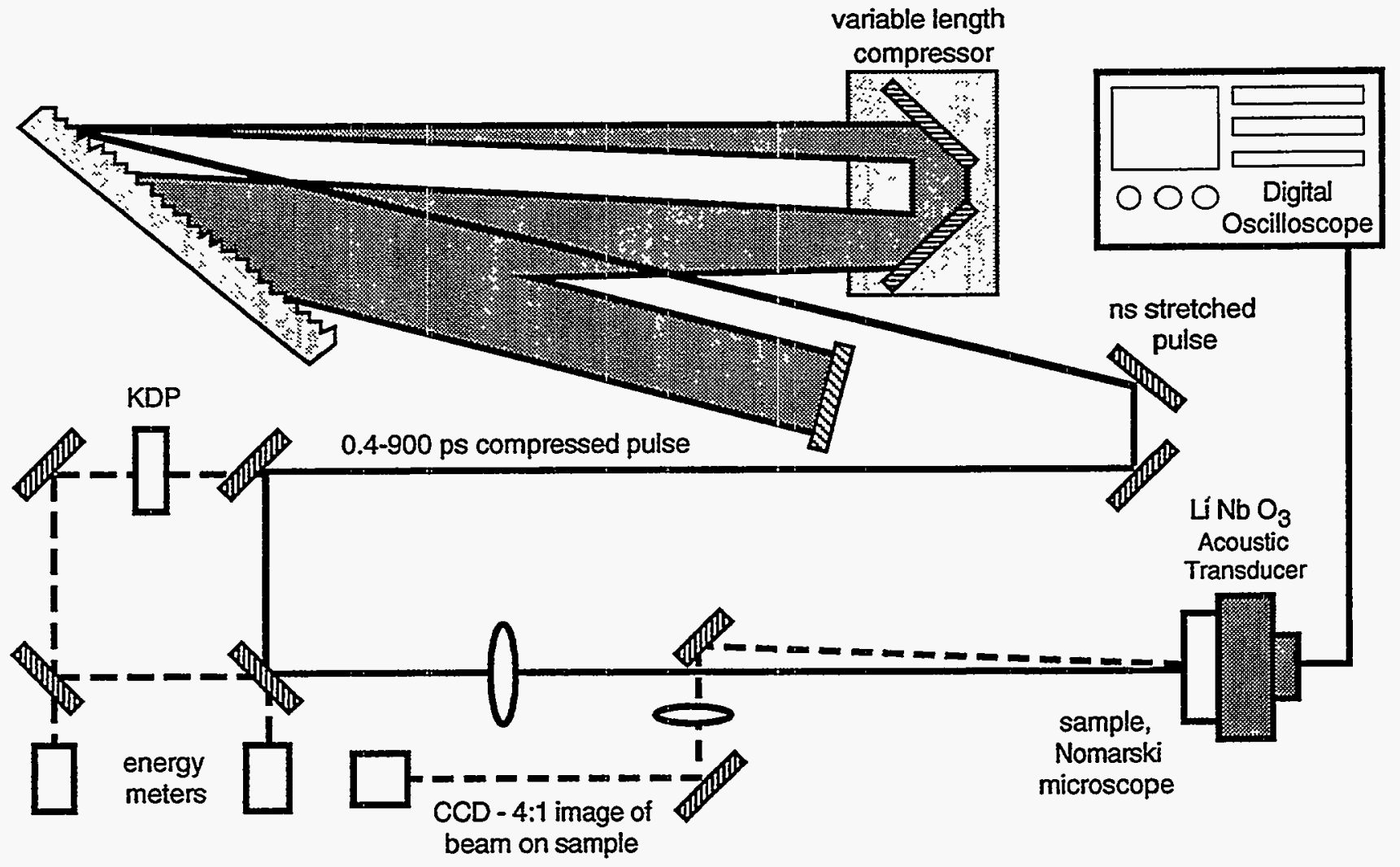

Fig. 1. Experimental set up: laser, tissue holder, transducer, microscope, scope, computer. 
After amplification, we compressed the pulses in a four-pass, single-grating (1740 line/mm) compressor of variable length. By varying the dispersive path length of the compressor, we obtained pulses of continuously adjustable duration from 300 fs to $1 \mathrm{~ns}$ (all reported pulsewidths are intensity fullwidth at half maximum). The temporal profile of the compressed pulses depends strongly on the spectral and temporal profile of the stretched pulse. For our experiments, we compressed a near Gaussian spectral profile to obtain temporally smooth tophat output pulses. This allowed us to relate the time evolution of the pulse intensity to the measured fluence.

The energy of each pulse was monitored with the leakage through a $92 \%$ reflectivity mirror. We adjusted the energy delivered to the damage sample with a half-waveplate before compression, using the strong dependence of grating efficiency upon input polarization. The RMS energy stability was typically less than $1.5 \%$, and we report the average value here. Due to saturated amplification in the regenerative amplifiers, the maximum energy never exceeded the average by more than $3 \%$.

We performed ablation measurements with laser spot size of $0.5 \mathrm{~mm}$ diameter ( $\mathrm{e}^{-2}$ intensity). Laser pulses were focused onto the tissue sample by a 1 meter focal length lens, with a variable distance to the sample. The spot size was measured with a CCD camera. The laser spatial mode at the sample had a $98 \%$ or better fit to a Gaussian, so the effective diameter as measured on the camera system was combined with the measured energy to give the pulse energy fluence. Our estimated absolute uncertainty in fluence was $15 \%$, but relative values should be within $5 \%$.

\subsection{Methods to study ablation parameters}

After irradiation, a Nomarski microscope was used to inspect the sample for possible formation of an ablated crater. We defined ablation threshold as any visible permanent removal of material from the sample surface observable with the Nomarski microscope. The smallest craters we could observe were approximately $1 \mu \mathrm{m}$ in diameter, a factor of 500 smaller in area than the laser spot size. Many fluence levels (10 to 20) were examined above and below the ablation threshold for a given pulsewidth in order to establish the threshold value.

To give further insight into the ablation process, laser-induced stress transients were recorded for each ablation event. A lithium niobate acoustic transducer with sensitivity of $9 \mathrm{mV} / \mathrm{bar}$ and ultrasonic frequency band of $300 \mathrm{MHz}$ (WAT-13, Science Brothers Inc., Houston, TX) was used for time-resolved detection of stress waves. The amplitude of the stress wave characterizes the efficiency of the laser energy deposition. The time integral of the transient stress yields the recoil momentum due to material ejection. To determine ablation efficiency, $\eta[\mu \mathrm{m} / \mathrm{pulse}]$, we have measured ablated crater depth and diameter after 10 to 100 laser pulses. The energy of each pulse was recorded. All ablation threshold measurements performed with the aid of optical microscopy were verified via comparison with transient stress profiles and magnitudes. That is, the formation of a crater was correlated with dramatic changes in both the amplitude and total momentum of the stress wave.

\section{THEORY OF OPTICAL BREAKDOWN}

Optical breakdown in transparent materials can be understood in terms of an electron avalanche in which conduction band electrons, oscillating in response to the laser field, gain thermal energy by scattering from phonons ${ }^{9-15}$. If an electron can achieve an energy equal to the band gap between bound and ionized states of the electron, subsequent impact ionization promotes another valence electron into the ionized (non-bound) state. The resulting avalanche, similar to that in gases, leads to an irreversible change in the bulk structure of the irradiated medium.

The theoretical picture of laser-induced damage to a substance is much simpler for ultrashort lasers pulses with duration of less than $10 \mathrm{ps}$ than for longer pulses. In this ultrashort pulse regime, intensities corresponding to breakdown produce electrons via multiphoton photoionization, and these seed electrons initiate the avalanche. Indeed, as the femtosecond regime is reached, breakdown intensities approach the limit in which photoionization alone is capable of producing electron densities high enough to cause damage or ablation. The strong nonlinear dependence of multiphoton absorption on intensity causes the threshold to become increasingly sharply defined for shorter pulse durations. Because there are no statistical fluctuations in the number of seed electrons, one may define the intrinsic damage threshold of the material. More importantly, for very short laser pulses, energy is absorbed by the electrons much faster than it is transferred to the lattice (atomic matrix of media). Since the lattice is not heated appreciably during the laser pulse, there is no modification of electron lattice scattering rate. There is also no need to track the flow of energy into the lattice to account for the thermomechanical stress during the pulse 
as is necessary for longer pulses, greater than $50 \mathrm{ps}$. The actual damage or medium ablation occurs after the ultrashort pulse has passed.

The evolution of free electron density $n$ can be described in terms of a simple rate equation ${ }^{13}$

$$
\frac{\partial n}{\partial t}=\beta I n+P(I)
$$

Here $I$ is the light intensity, $P(I)$ is the rate of multiphoton ionization, and $\beta$ describes the avalanche rate $(\beta=11$ $\mathrm{cm}^{2} / \mathrm{TW} / \mathrm{s}$ ). For $1053 \mathrm{~nm}$ light, six-photon absorption cross section values for water were not available, so we used the strong field Keldysh ${ }^{14}$ formulae for $P(I)$. The only parameter of importance for this expression is the bandgap between the ground electronic state of valence electron and the ionized state of the electron. We used the value of $6.5 \mathrm{eV}$, typical for distilled water ${ }^{15.16}$. Evaluation of the Keldysh expression leads to a result that fits nicely to the six-photon absorption form, $P(I)=C I^{6}$, where $\mathrm{C}=1.599 * 10^{16}\left(\mathrm{~cm}^{2} / \mathrm{TW}\right)^{6} / \mathrm{s}$.

For calculation of the avalanche rate on the surface of biological tissue, one must have detailed information about the electron scattering rate there. Such data are unavailable in the literature, so we used the rate calculated for glass ${ }^{13}$. Due to the amorphous structure of both materials, the actual value for aqueous media should be close to that of glass.

We assume that optical breakdown threshold is indicated by the occurrence of a sufficiently high free electron density. A reasonable lower limit would be on the order of $10^{19} / \mathrm{cc}$, roughly the density at which the energy density of ionized electrons equals the binding energy of the lattice. A more realistic choice for the electron density at the breakdown threshold is the critical electron density at which the plasma becomes reflective $\left(10^{21} / \mathrm{cc}\right.$ for $\left.1053 \mathrm{~nm}\right)$. Such a density is just below the density at which laser pulse is strongly absorbed. Our calculations indicate that the theoretical threshold is only a logarithmic function of electron density.

It is natural to relate the linear absorption coefficient in media to the number of initial free electrons. We calculated the number of initial electrons using the same scattering rate as was used for the avalanche rate calculation. Damage threshold calculations for materials with various linear absorpbances are presented in Fig. 2. One can see that the threshold becomes insensitive to the linear absorption when laser pulse becomes very short. This is because multiphoton ionization produces many more free electrons than initially present or produced by linear heating.

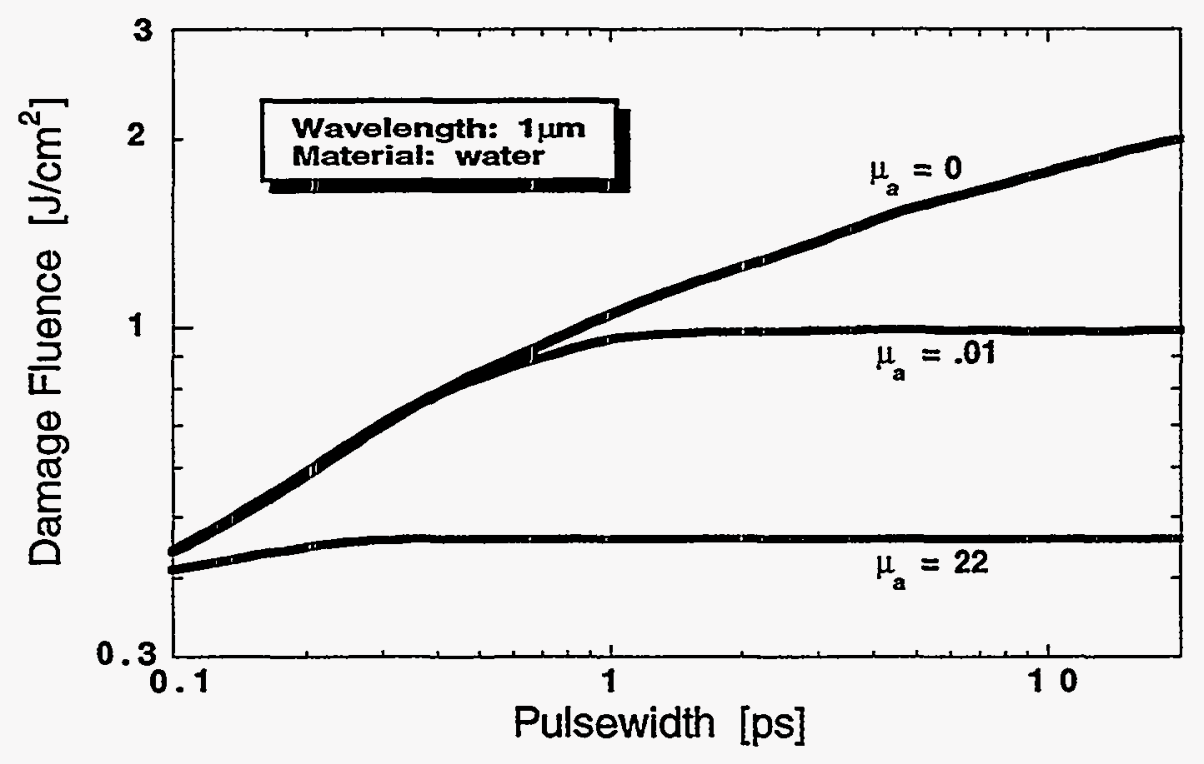

Fig. 2. Theoretical curve of plasma breakdown threshold as a function of pulse duration 
Next, we consider the distribution of deposited laser energy. The laser light energy is deposited in the electrons produced by the avalanche and multiphoton ionization. Hence, we can express the axial intensity distribution in a medium as

$$
\frac{\partial I}{\partial z}=-\varepsilon \frac{\partial n}{\partial t}
$$

where the electron density rate, $\partial n / \partial t$, is described by Eq. (1), $\varepsilon$ is the average energy of electrons including the ionization energy $E_{i}$ from the ground state. It was shown ${ }^{13}$ that this total energy can be estimated as $1.5 \times E_{i}$ or about $10 \mathrm{eV}$. Solving Eqs. (1) and (2) for different pulse durations and pulse energies close to the optical breakdown threshold values leads to the results presented in Fig. 3. One can see that the absorbed energy is localized in a thin layer of about only $1 \mu \mathrm{m}$ thick (i.e., the effective absorption coefficient $\mu_{a}^{\prime}=7000 / \mathrm{cm}$ ) and is practically independent of the laser pulse duration. As a result of multiphoton absorption one can observe a slowly decreasing long energy deposition tail after the initial very fast decay. This can yield relatively deep penetration of low intensity background prepulses in the case of ultrashort pulse irradiation.

If the pulse energy exceeds the threshold, the electron density near the surface becomes larger than the critical value $n_{c}$ and a plasma mirror is formed, which means that the laser radiation cannot penetrate into the tissue. Nevertheless, within the skin depth laser radiation produces more free electrons. These new free electrons continue to be relatively cold due to losses of laser energy for ionization. High plasma density and low electron energy result in high absorptance (of $50 \%$ and up) within the skin depth. With the increase of the electron density the skin depth decreases. When all the electrons are ionized, the skin depth can be calculated as

$$
\delta=\frac{\lambda}{2 \pi \sqrt{n_{s} / n_{c}}}
$$

where $n_{s}$ stands for solid state density. After all of the electrons are ionized energy absorption results in electron heating and a fast drop of the absorption efficiency. This is an indication that an optimal energy for plasma mediated tissue ablation exists in case of ultrashort laser pulses. Experimental determination of the optimum energy has yet to be done.

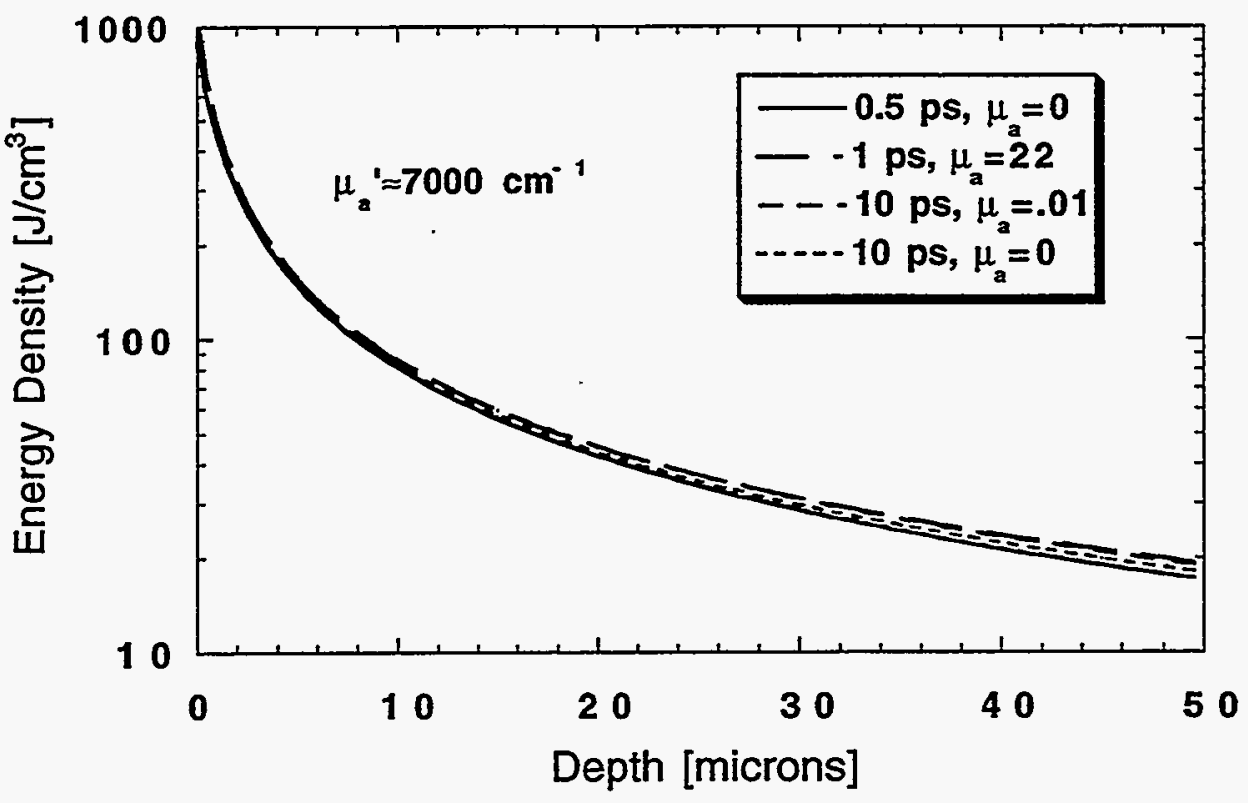

Fig. 3. Effective absorbence in materials for various pulse durations. 
At the end of the laser pulse, the absorbed energy is transferred to the lattice on the time scale of picoseconds. The laser energy density at the breakdown threshold on the tissue surface is well above the molecular binding energy. Therefore, the tissue within the heated layer can be considered as a gas. The subsequent process of material ejection and crater formation has a universal nature and is independent of details of the tissue structure and molecular composition.

\section{EXPERIMENTAL RESULTS}

Figure 4 presents the results of abiation threshold measurements for collagen gels dyed to have different linear absorption and porcine cornea. Notice that the ablation thresholds for corneas and collagen gels follow the trends predicted by optical breakdown thresholds predicted in the previous section. For absorbing gels, nanosecond laser ablation produced no noticeable plasma. However, with the decrease in pulse duration, more and more noticeable plasma was detected. Picosecond ablation was unavoidably mediated by plasma independent of material properties. The ablation threshold for porcine cornea in the nanosecond pulsewidth range $\left(75 \mathrm{~J} / \mathrm{cm}^{2}\right)$ is about 1000 times higher than the ablation threshold for black gel $\left(0.074 \mathrm{~J} / \mathrm{cm}^{2}\right)$. In the femtosecond pulsewidth range this difference decreases to only a factor of 6 . Thus, the ablation thresholds for transparent and nontransparent tissues converge at shorter pulsewidths. Ablation thresholds for transparent comea can be described approximately as a square root function of laser pulse duration with a deviation in the femtosecond range. Similar findings were reported by Puliafito et al. for photodisruption of human corneas ${ }^{1}$. The ablation threshold for black gel is practically independent of laser pulse duration.

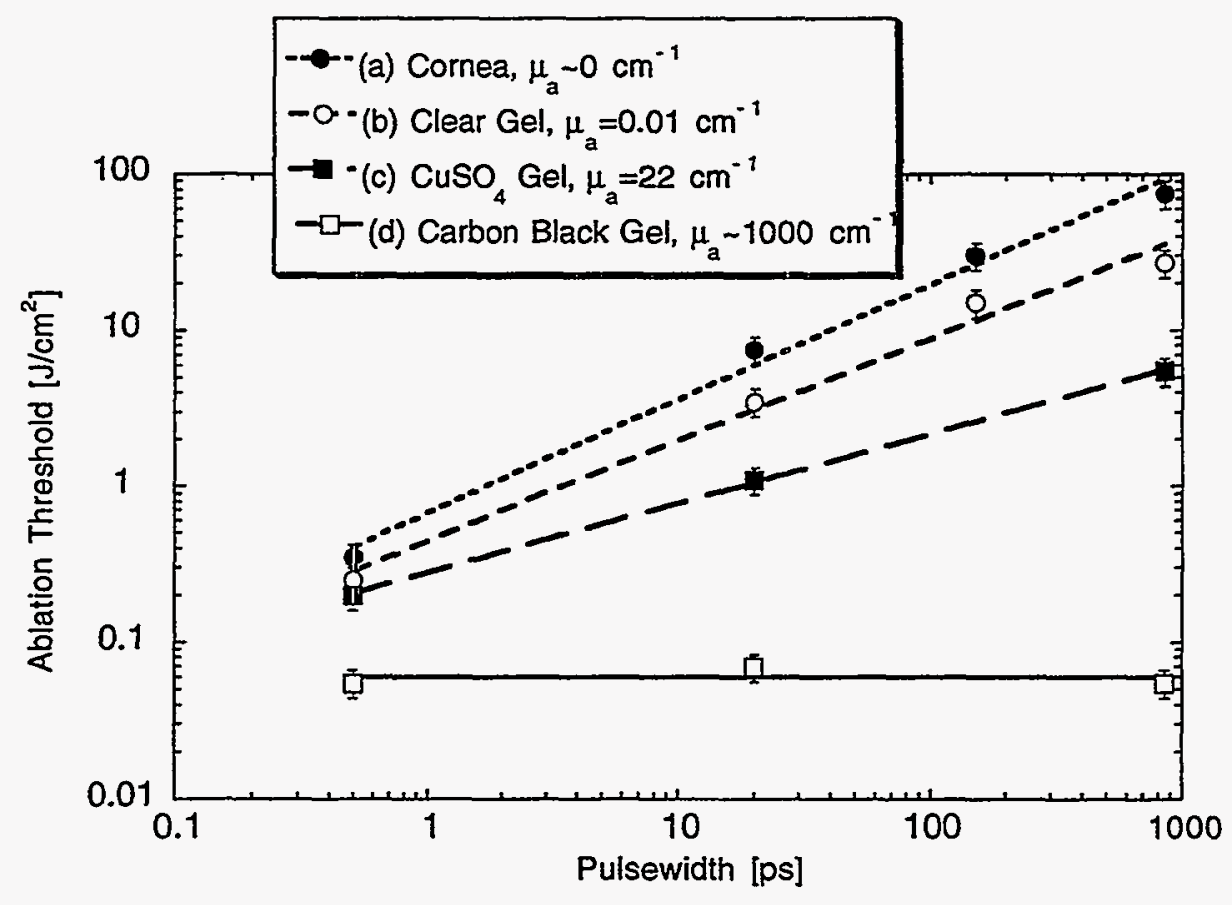

Fig. 4. Ablation threshold fluence as a function of laser pulse duration for various materials: (a) porcine comea $\left(\mu_{a}=0\right)$, (b) clear collagen gel with no cupric chloride $\left(\mu_{a}=0.01 / \mathrm{cm}\right)$, (c) collagen gel with cupric chloride $\left(\mu_{a}=22 / \mathrm{cm}\right)$, and (d) collagen gel with high concentration of carbon black $\left(\mu_{a}=1000 / \mathrm{cm}\right)$. Laser fluence was gradually increased until the moment when a microscopically detectable crater was observed after 10 laser pulses. 


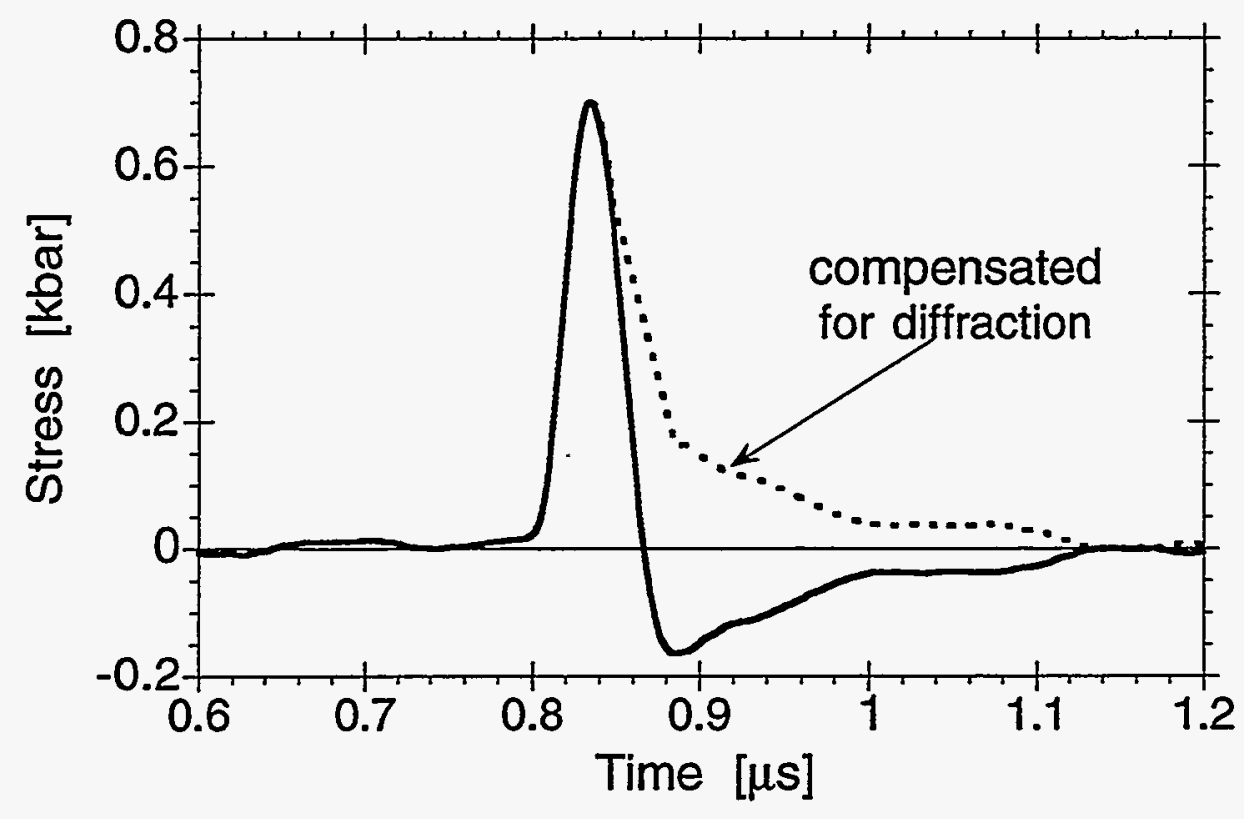

Fig. 5. Stress transient induced in collagen gel $\left(\mu_{a}=76 / \mathrm{cm}\right)$ by $350 \mathrm{fs}$ pulses at $1053 \mathrm{~nm}$ with a fluence of $0.37 \mathrm{~J} / \mathrm{cm}^{2}$ (about 3 times the ablation threshold). Pressure magnitudes were normalized by the factor $\mathrm{R} / \mathrm{r}$ where $\mathrm{R}$ is the distance at which the stress was detected and $\mathrm{r}$ is the radius of the laser beam.

Figure 5 presents the stress transient measured by the lithium niobate acoustic transducer due to femtosecond laser ablation of $\mathrm{CuCl}_{2}$ colored gel. The negative stress detected by the piezoelectric transducer is an artifact associated with the diffraction of ultrasonic waves ${ }^{17}$. The laser beam, focused to a spot of diameter $350 \mu \mathrm{m}$, produces a spherical wave at the surface of piezoelectric element, located at the distance of $6.95 \mathrm{~mm}$ relative to the irradiated surface. The effect of a spherical wave on our relatively wide detector ( $3 \mathrm{~mm}$ diameter) is to simulate a "false" negative stress. When one corrects for the diffraction effect, the acoustic signal has the profile shown by the dashed line. The maximal amplitude of the recorded transient shock wave is about $700 \mathrm{bar}$, and the maximal shock gradient is $30 \mathrm{bar} / \mathrm{ns}$ or $19 \mathrm{bar} / \mathrm{\mu m}$.

Figure 6 demonstrates the acoustic transducer signal due to a 200 ps irradiation of a collagen gel. Low fluence irradiation $\left(0.1 \mathrm{~J} / \mathrm{cm}^{2}\right)$ with a wide laser beam has induced approximately symmetrical stress transient with an amplitude of approximately 6 bar. A plasma was not observed in this case. High fluence irradiation $\left(8.5 \mathrm{~J} / \mathrm{cm}^{2}\right)$ produced optical breakdown on the surface of gel and subsequent plasma mediated ablation. This fluence was about 3 times higher than the ablation threshold for the collagen gel. In contrast to 350 fs pulse ablation (Fig. 5), the plasma mediated ablation by a 200 ps pulse has given rise to a shock wave of more than $20 \mathrm{kbar}$ amplitude. The pressure of $21 \mathrm{kbar}$ was calculated based on spherical spreading of the ultrasonic wave. Such a strong shock propagating through the tissue could produce a substantial mechanical damage to the medium around ablation crater. Even after attenuation of higher ultrasonic frequencies by the gel and quartz the detected shock front has a maximal gradient of $150 \mathrm{bar} / \mathrm{ns}$ which corresponds to $100 \mathrm{bar} / \mu \mathrm{m}$. Note that because of the nonlinear propagation of high amplitude acoustic waves, the arrival time of the high amplitude stress wave at the acoustic detector is $440 \mathrm{~ns}$ earlier then the arrival of the low amplitude stress wave. 


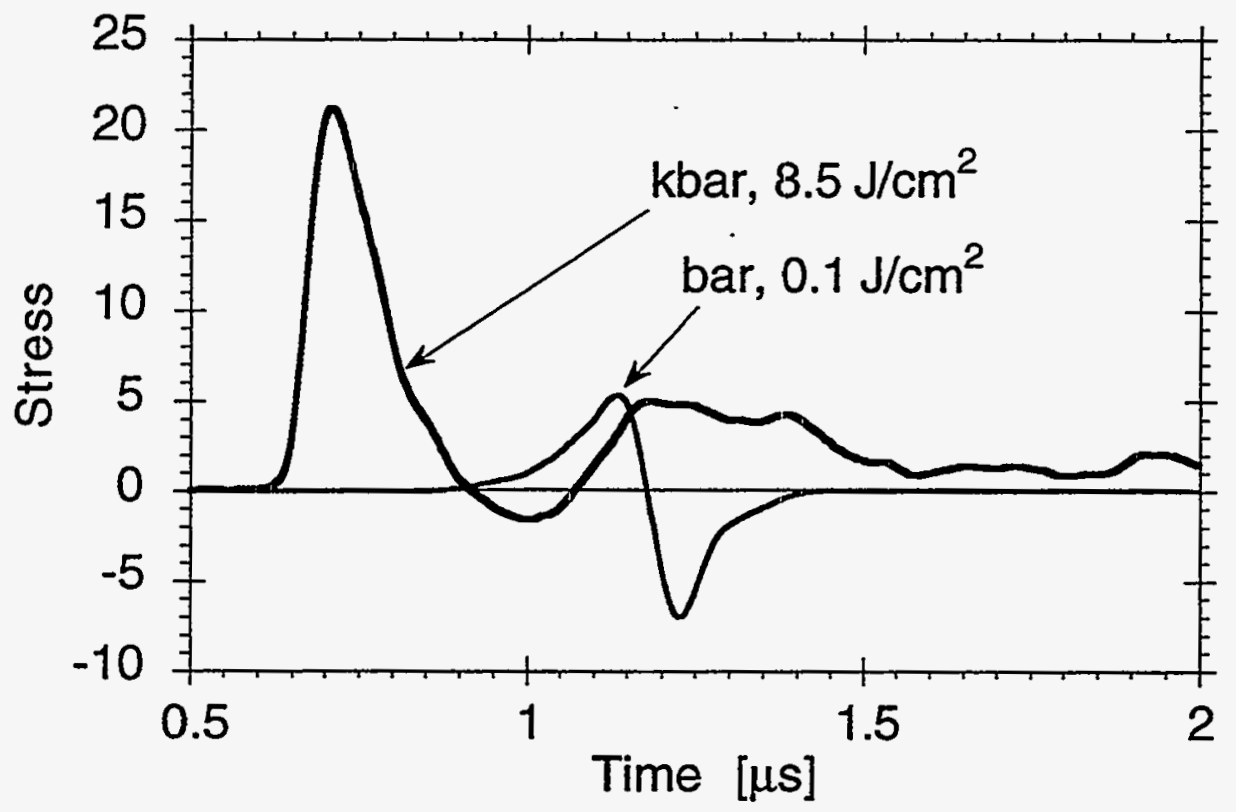

Fig. 6. Stress transients induced in collagen gel $\left(\mu_{\mathrm{a}}=76 / \mathrm{cm}\right)$ by a 200 ps puise of a Nd:YAG laser.

Figure 7 illustrates typical craters produced by nanosecond and subpicosecond pulses. There appears to be much more thermal damage for the longer nanosecond pulses. For ultrashort pulses, collateral damage is practically absent. A clean and smooth crater is produced as shown in the left part of Fig. 7a. The ablation rate of 6 to $8 \mu \mathrm{m} / \mathrm{pulse}$ is reasonably insensitive to the initial linear absorption coefficient, the pulse width and the laser fluence provided that the fluence is greater than a few times the ablation threshold.

(a)

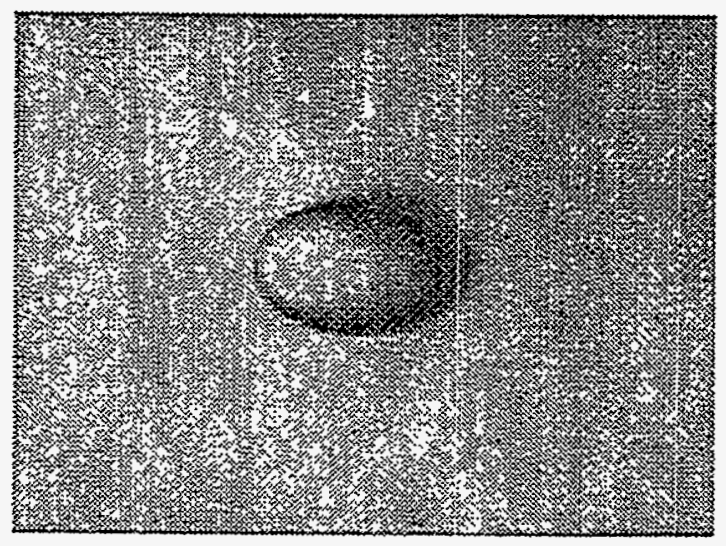

(b)

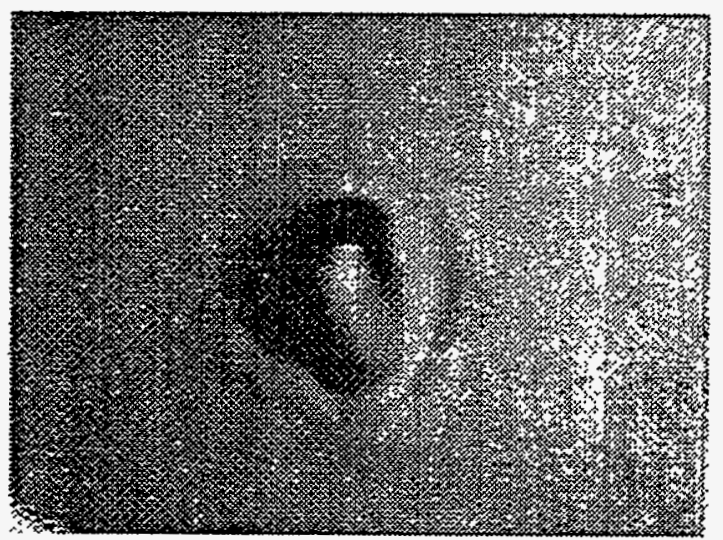

Fig. 7. Craters produced in a clear collagen gel by plasma mediated ablation with one pulse of a Ti:sapphire laser at $1053 \mathrm{~nm}$. Parameters of irradiation: (a) $350 \mathrm{fs}, 2.3 \mathrm{~J} / \mathrm{cm}^{2}$; and (b) $1 \mathrm{~ns}, 21 \mathrm{~J} / \mathrm{cm}^{2}$ laser pulses. 


\section{DISCUSSION AND CONCLUSIONS}

We explored, experimentally and theoretically, the process of tissue ablation by ultrashort pulses. Several aspects of the ablation could be helpful in cases were precise tissue ablation is desired. First, the ablation threshold becomes insensitive to material properties as the pulse length decreases into the subpicosecond regime. Second, the ablation crater has sharp edges and a smooth bottom for subpicosecond pulses. Third, for all pulse lengths and materials studied, the ablation rate is always about 6 to $8 \mu \mathrm{m}$ per pulse provided that the laser fluence is several time the ablation threshold. Fourth, the crater diameter does not exceed the laser beam diameter.

These results are still preliminary. Further research needs to be done to understand what happens to the energy after it is deposited by the laser. How the energy couples to the lattice, how the tissue is ablated, how the stress wave is generated, how the stress wave propagates into the tissue, and how the collateral damage occurs all warrant further investigation.

\section{ACKNOWLEDGMENTS}

We would like to thank Steve Herman for invaluable technical assistance. This work was performed under the auspices of the US Department of Energy by the Lawrence Livermore National Laboratory under contract W-7405-ENG-48. Authors would also like to acknowledge partial support provided by The Whitaker Foundation.

\section{REFERENCES}

1. C.A. Puliafito, R. Birngruber, T.F. Deutsch, J.G. Fujimoto, D. Stern, and B. Zysset, "Laser-Tissue Interactions in the nanosecond, picosecond and femtosecond time domains", Photoacoustic and Photothermal Phenomena II, ed. by IC Murphy et al., Springer Series in Optical Sciences, Vol. 62, Springer-Verlag, Berlin, pp. 420-427, 1990.

2. F. Docchio, C.A. Sacchi, and J. Marshall, "Experimental investigation of optical breakdown thresholds in ocular media under single puise irradiation with different pulse durations", Lasers in Ophthalmology, Vol. 1, pp. 83-93, 1986.

3. R. Birngruber, C.A. Puliafito, A. Gawande, W.-Z. Lin, R.T. Schoenlein, and J.G. Fujimoto, "Femtosecond LaserTissue interactions: Retinal injury studies", IEEE J. Quantum Electron., Vol. QE-23, p. 1836, 1987.

4. B. Zysset, J.G. Fujimoto, C.A. Puliafito, R. Bimgruber, and T.F. Deutsch, "Picosecond optical breakdown: Tissue effects and reduction of collateral damage", Lasers Surg. Med., Vol. 9, pp. 193-204, 1989.

5. A. Vogel, S. Busch, K. Jungnickel, R. Birngruber, "Mechanisms of intraocular photodisruption with picosecond and nanosecond laser pulses", Lasers Surg. Med., Vol. 15, pp. 32-43, 1994.

6. N.F. Bunkin and F.V. Bunkin, "The concepts in the optical breakdown of transparent liquids", Laser Physics, Vol. 3, pp. 63-77, 1993.

7. B.C. Stuart, M.D. Feit, S. Herman, A.M. Rubenchik, B.W. Shore, and M.D. Perry, "Nanosecond to femtosecond laser-induced breakdown in dielectrics", Phys. Rev. B, in press, 1995.

8. B.C. Stuart, S. Herman, and M.D. Perry, "Laser system with tunable pulse-width", IEEE J. Quantum Electron., in press, 1995.

9. S.C. Jones, P. Braunlich, R.T. Casper, X.-A. Shen, and P. Kelly, Opt. Eng., Vol. 28, p. 1039, 1989.

10. N. Bloembergen, IEEE J. Quantum Electron., Vol. QE-10, p. 375, 1974.

11. A.A. Manenkov and A.M. Prokhorov, Sov. Phys. Usp. Vol. 29, p. 107, 1986.

12. M. Sparks, D.L. Mills, R. Warren, T. Holstein, A.A. Maradudin, L.J. Sham, E. Loh Jr., and D.F. King, Phys. Rev. B, Vol. 24, p. 3519, 1981.

13. B.C. Stuart, M.D. Feit, A.M. Rubenchik, B.W. Shore, and M.D. Perry, Phys. Rev. Letters, in press, 1995.

14. L.V. Keldysh, Sov. Phys., JETP, Vol. 20, p. 1307, 1965.

15. C.A. Sacchi, J. Opt. Soc. Am. B, Vol. 8, p. 337, 1991.

16. D.N. Nikogosyan and A. A. Oraevsky, V.I. Rupasov, "Two-photon ionization and dissociation of liquid water by powerful laser UV radiation", Chem Phys, Vol. 77, pp. 131-143, 1983.

17. V.E. Gusev and A.A. Karabutov, Laser Optoacoustics, American Institute of Physics (AIP Press), New York, pp. 27-34, 1994. 